\title{
Fetal and early life growth and body mass index from birth to early adulthood in 1958 British cohort: longitudinal study
}

Tessa J Parsons, Chris Power, Orly Manor

\begin{abstract}
Objectives To determine the influence of birth weight on body mass index at different stages of later life; whether this relation persists after accounting for potential confounding factors; and the role of indicators of fetal growth (birth weight relative to parental size) and childhood growth.

Design Longitudinal study of the 1958 British birth cohort.

Setting England, Scotland, and Wales.

Participants All singletons born 3-9 March 1958

(10 683 participants with data available at age 33).

Main outcome measures Body mass index at ages 7 , $11,16,23$, and 33 years.

Results The relation between birth weight and body mass index was positive and weak, becoming more J shaped with increasing age. When adjustments were made for maternal weight, there was no relation between birth weight and body mass index at age 33 . Indicators of poor fetal growth based on the mother's body size were not predictive, but the risk of adult obesity was higher among participants who had grown to a greater proportion of their eventual adult height by age 7 . In men only, the effect of childhood growth was strongest in those with lower birth weights and, to a lesser extent, those born to lighter mothers. Conclusions Maternal weight (or body mass index) largely explains the association between birth weight and adult body mass index, and it may be a more important risk factor for obesity in the child than birth weight. Birth weight and maternal weight seem to modify the effect of childhood linear growth on adult obesity in men. Intergenerational associations between the mother's and her offspring's body mass index seem to underlie the well documented association between birth weight and body mass index. Other measures of fetal growth are needed for a fuller understanding of the role of the intrauterine environment in the development of obesity.
\end{abstract}

\section{Introduction}

Intrauterine life is a critical period for the development of obesity later in life. 'Growth in utero is summarised, albeit crudely, by birth weight, which, if related to fatness later in life, might implicate the fetal environment in the development of obesity. The relation between birth weight and fatness, measured in childhood or adulthood, is generally positive, although it is variable in magnitude. ${ }^{23}$ A possible reason for this variability is that the strength of the relation may depend on the age at which fatness is measured. More importantly, several factors, such as gestational age, parental body size, and socioeconomic status, may confound the relation between birth weight and later fatness. Few studies have attempted to account for these factors, ${ }^{23}$ and our under- standing of the relation remains limited. Some studies report a $\mathrm{J}$ shaped or $\mathrm{U}$ shaped relation, with a higher prevalence of obesity seen for the lowest and highest birth weights, ${ }^{4-8}$ suggesting a more complex association between growth in utero and obesity. One possibility is that birth weight does not adequately reflect the effect of intrauterine environment on growth, and other indicators may be more appropriate. A potentially useful approach is to examine birth weight relative to genetic potential, indicated by the size (height, weight, or body mass index (weight $(\mathrm{kg}) /\left(\right.$ height $\left.(\mathrm{m})^{2}\right)$ ) of the parents or by the individual's adult body height.

Failure to realise growth potential has previously been found to be more strongly related to high blood pressure than to birth weight. ${ }^{9}$ Other studies implicate faster, earlier childhood growth in the development of obesity, ${ }^{10-12}$ yet few studies have tried to assess how patterns of growth in utero and in childhood affect the risk of obesity. The aims of this study were to establish whether birth weight is related to subsequent body mass index at different life stages (from childhood to early adulthood), whether the relation between birth weight and adult body mass index at age 33 persists after accounting for potential confounding factors, and whether indicators of fetal growth, represented by a combination of birth weight and parental size, adult height, or growth in childhood, are related to adult obesity.

\section{Participants and methods}

We used data from the 1958 British birth cohort, which included all children born in England, Scotland, and Wales in the week of 3-9 March 1958. ${ }^{13}{ }^{14}$ Information was obtained on $17414(98.2 \%)$ births of the target population (17 733 births). Major follow ups of surviving children were conducted at ages $7,11,16,23$, and 33 years. $^{15}$ At age 33, $11407(72.8 \%)$ participants from a target sample of 15667 subjects provided information. We excluded multiple births $(n=446)$ from all analyses. Attrition of the sample has resulted in a slight under-representation of those participants who are most disadvantaged, but the remaining sample is generally representative of the original sample. ${ }^{15}$ The present study is largely concerned with participants with data available about body mass index at age 33 . Mean body mass index and associations between birth weight and body mass index at each age in the subset were similar to those for the larger dataset. For example, when we compared the sample with data at age $33(n=10683)$ with the sample with data at age $7(n=12985)$, men who had data at age 33 had a mean body mass index of 15.94 (SD 1.58) $\mathrm{kg} / \mathrm{m}^{2}$ at age 7 , and all those with data at age $7 \mathrm{had}$ a mean body mass index of $15.95(1.63) \mathrm{kg} / \mathrm{m}^{2}$ at age 7 . For women, mean body mass index at age 7 was 15.86 (1.89) $\mathrm{kg} / \mathrm{m}^{2}$ for those with data at age 33 , and 15.89 (1.91) $\mathrm{kg} / \mathrm{m}^{2}$ for all those with data at age 7 .
Editorial by Law

Department of Paediatric

Epidemiology and Biostatistics, Institute of Child

Health, London

WC1N 1EH

Tessa J Parsons

research fellow

Chris Power

reader

School of Public

Health and

Community

Medicine, Hebrew

University,

Jerusalem 91120 ,

Israel

Orly Manor

senior lecturer

Correspondence to: T Parsons

t.parsons@ich.ucl.

ac.uk

BMJ 2001;323:1331-5 


\section{Body mass index}

At ages 7, 11, and 16, heights (to the nearest inch) and weights (in underclothes, to the nearest pound) were measured by trained medical personnel. At age 23, weight and height were reported by the participants themselves. At age 33, height was measured to the nearest centimetre, and weight was measured, with indoor clothing but without shoes, to the nearest $0.1 \mathrm{~kg}$. Data at ages 23 and 33 were checked to detect coding errors. ${ }^{16} 17$ Body mass index values for women who were pregnant at age $33(n=256)$ were excluded. Obesity at age 33 was defined as body mass index $\geqslant 30 .^{18}$

\section{Fetal and early life growth}

Birth weights were recorded in pounds and ounces by the midwives in charge of the deliveries, and we converted the values to grams. Growth in utero was also expressed as birth weight (in three groups divided by tertiles) relative to parental size--height, weight, or body mass index-or to the individual's own subsequent growth-height at age 33 and the percentage of adult height achieved by age 7 (given as (height at age $7 /$ height at age 33$) \times 100$ ).

\section{Potential confounding factors}

Gestational age, parental fatness, socioeconomic status, maternal smoking during pregnancy, parity, and mother's age at the time of birth were identified from the literature as potential confounding factors.

Gestational age: Gestational age was the number of days from the date of the first day of the mother's last menstrual period.

Parental body size: Maternal height without shoes was measured, and weight before pregnancy was reported by the mother, in categories of one stone, shortly after the birth of the cohort member. In 1969, when the child was 11 years old, the heights and weights of both parents were reported by the mother. Height was reported to the nearest inch, and weights were classified into one of 27 groups ranging from 6 stone 4 pounds $(39.9 \mathrm{~kg})$ to 19 stone 10 pounds $(125.2$ $\mathrm{kg})$. To estimate body mass index, parents were assigned a weight equivalent to the midpoint of their weight group.

Socioeconomic status: Social class was defined in terms of the father's occupation according to the 1951 registrar general's classification. ${ }^{19}$ We used five categories in our analyses-classes I and II (professional and managerial), III-NM (skilled non-manual), III-M (skilled manual), IV and V (semi-skilled and unskilled manual), and those recorded as having "no male head of household."

Maternal smoking: Maternal smoking after the fourth month of pregnancy was reported shortly after

Table 1 Birth weight and body mass index of members of the 1958 British birth cohort at $7,11,16,23$, and 33 years

\begin{tabular}{lcclcc} 
& \multicolumn{2}{c}{ Male } & & \multicolumn{2}{c}{ Female } \\
\cline { 2 - 3 } \cline { 6 - 6 } & No & Mean $($ SD) & & No & Mean (SD) \\
\hline Birth weight $(\mathrm{g})$ & 8429 & $3380(0.57)$ & & 7954 & $3250(0.55)$ \\
\hline Body mass index $\left(\mathrm{kg} / \mathrm{m}^{2}\right):$ & & & & \\
\hline 7 years & 6717 & $15.95(1.63)$ & & 6268 & $15.89(1.91)$ \\
\hline 11 years & 6233 & $17.31(2.41)$ & & 5982 & $17.66(2.71)$ \\
\hline 16 years & 5577 & $20.25(2.73)$ & & 5233 & $21.02(2.96)$ \\
\hline 23 years & 5999 & $23.11(2.91)$ & & 5599 & $22.07(3.18)$ \\
\hline 33 years & 5375 & $25.63(4.01)$ & 5308 & $24.60(4.87)$ \\
\hline
\end{tabular}

the cohort member's birth, and the mother was categorised as a non-smoker $(<1$ cigarette per day) or smoker ( $\geqslant 1$ cigarette per day).

Maternal age and parity: The numbers of previous births (live births and stillbirths after 28 weeks' gestation) were reported in 1958. Maternal age was treated as a continuous variable, and parity as dichotomous-first child or not first child. Maternal diabetes during pregnancy was recorded in 1958.

\section{Statistical analyses}

All analyses were performed using SPSS and were carried out separately for male and female cohort members. The association between birth weight and body mass index was investigated using linear regression models, and linearity was tested using a quadratic term for birth weight. In all cases (except for men at ages 16 and 33 and women at age 7 ), the quadratic term was significant, indicating non-linearity. We therefore inspected the relation using alternative centile groups to identify a categorisation that would best represent the shape of the relation. Accordingly, birth weight was expressed for each sex separately, in fifths or, where fifths were impractical for presentation, in thirds. Since the variance of body mass index changes with age, we calculated sex specific standard deviation scores for body mass index for each subject to compare the relation between birth weight and body mass index at each age. In further analyses of body mass index at age 33 , we examined the effect of several potential confounding factors on the relation between birth weight and adult body mass index. Although all factors (except father's body mass index) were significantly related to birth weight in both sexes $(\mathrm{P}<0.05)$, several factors (gestational age, father's height, and mother's age and parity) were unrelated to body mass index at age 33 , and they were excluded from further analyses. Only 20 mothers were recorded as having diabetes during pregnancy; adjustment for maternal diabetes made no difference to the findings. Additional analyses showed that, when included simultaneously in a linear model, parent's weight and height had a similar effect as their body mass index on their child's body mass index at age 33 (data not shown). Maternal weights and heights recorded in 1958 were used rather than those taken in 1969 in order to include a larger sample; results were similar for data from both dates.

\section{Role of fetal growth}

The role of fetal growth was investigated by examining the relation between birth weight and obesity for different levels of paternal or maternal body size or for the cohort member's adult height. Poor fetal growth would be implicated if the risk of obesity was increased in children who had a lower birth weight than expected (given their mother's or father's heights, weight, or body mass index) or the height they achieved as an adult. This was tested with an interaction term between parental body size (or the adult height of the cohort member), birth weight, and obesity at age 33 in a logistic regression model, using obesity at age 33 as the dependent variable. We also examined the effect of the percentage of adult height achieved by age 7 on adult obesity for different levels of birth weight.

All independent variables except birth weight were treated as continuous, but for presentation purposes they are displayed as fifths or thirds. The distribution of body mass indices showed a slightly positive skew, so 
analyses were repeated after we transformed variables to natural logarithms; the results were similar (data not presented).

\section{Results}

Mean birth weight and body mass index are given in table 1 . At each age, body mass index increased with increasing birth weight (table 2). As age increased, the difference in mean body mass index, as determined by sex specific standard deviation scores, between the lower and middle thirds of birth weight disappeared, while the difference between the lower and upper thirds fell but remained significant through to age 33 . The shape of the relation changed with age, from a linear shape to a J shape in women and tending towards a J shape in men. The association was weak: in men $1.7 \%$ of the variance (adjusted $R^{2}$ ) was explained at age 7 , falling to $0.3 \%$ at age 33 , with similar proportions seen in women.

In adulthood, body mass index increased with increasing birth weight mainly at the heaviest birth weights (fig 1). In men, an increase in birth weight of $0.5 \mathrm{~kg}$ from $3.7 \mathrm{~kg}$ to $4.2 \mathrm{~kg}$ resulted in an estimated increase of $0.4 \mathrm{~kg} / \mathrm{m}^{2}$ in body mass index at age 33 , from $25.8 \mathrm{~kg} / \mathrm{m}^{2}$ to $26.2 \mathrm{~kg} / \mathrm{m}^{2}$. The relation between birth weight and body mass index was unaffected by the mother's height (fig 2), age, or smoking habits, father's weight, or social class (data not shown). The mother's weight and body mass index had a marked influence; when mother's weight was adjusted for, the difference between mean body mass index for the top and bottom fifths of birth weight fell from 0.72 to 0.28 in men and from 0.77 to 0.13 in women, and was no longer significant.

To further investigate the role of intrauterine growth, we examined the relation between measures of parental body size (height, weight, or body mass index), birth weight, and obesity among cohort members at age 33 using maternal body mass index as an example (fig 3). There was a weak relation between birth weight and adult obesity, which was similar for each third of maternal body mass index (the interaction term of birth weight by mother's body mass index on obesity at age 33 was not significant; $\mathrm{P}>0.05$ ). The risk of obesity was not increased in children whose mothers had a high body mass index but who had a low, rather than high, weight at birth. Results were not affected when body mass index at age 33 was used as a continuous outcome or when alternative indicators of genetic potential-father's body mass index, mother or father's height or weight, mean parental height, or the height of the cohort member at age 33 (data not shown)were used. These findings provide little evidence for a relation between growth in utero and adult obesity.

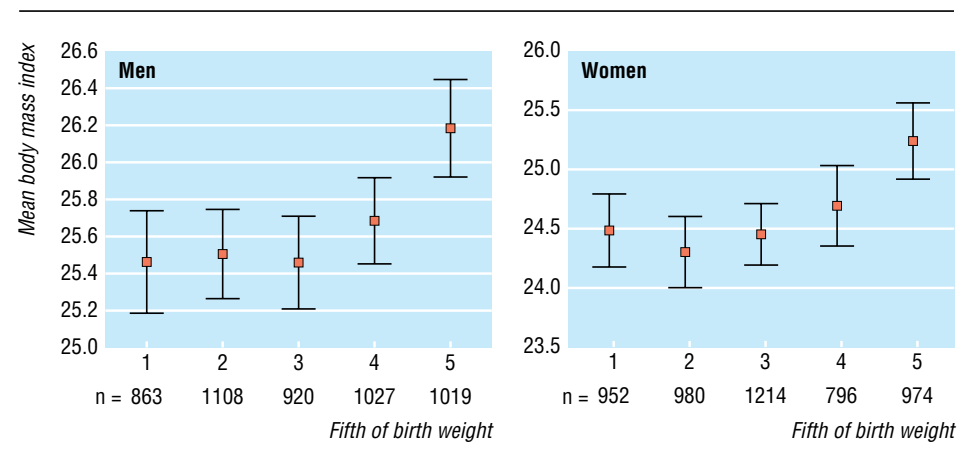

Fig 1 Relation between birth weight (fifths; lowest on left) and mean body mass index at age 33 in the 1958 British birth cohort. Bars indicate 95\% confidence intervals. Cut offs for quintiles 2950, 3290, 3520, and $3830 \mathrm{~g}$ in men and 2860, 3150, 3400, and $3660 \mathrm{~g}$ in women

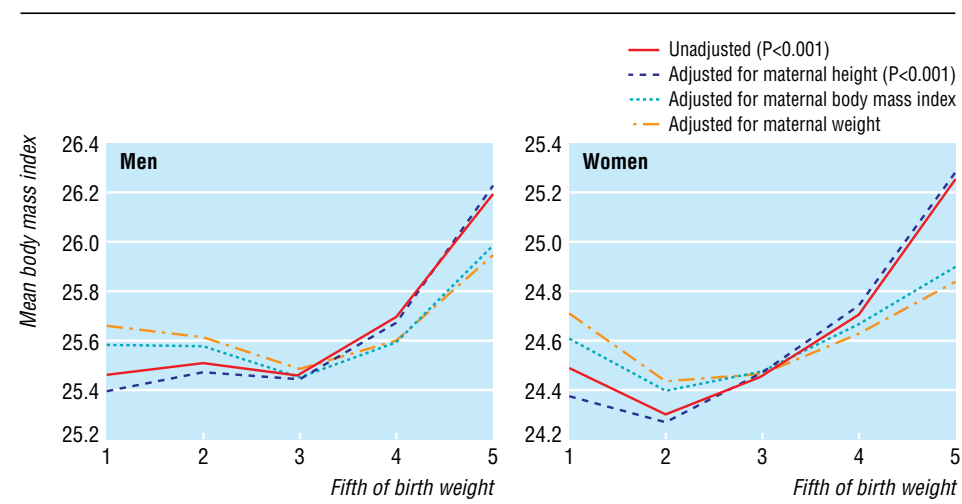

Fig 2 Influence of maternal height, weight, and body mass index on the relation between birth weight (fifths; lowest on left) and mean body mass index at age 33 in the 1958 British birth cohort. $P$ values for comparison of mean body mass index between lowest and highest fifths of birth weight

Childhood growth was related to adult obesitycohort members who achieved a greater percentage of their adult height by age 7 tended to be heavier rather than lighter at birth (data not shown) and had an increased risk of obesity at age 33 (fig 4). When we examined birth weight and subsequent childhood growth simultaneously, we found that the effect of childhood growth on adult obesity varied by birth weight in men, with a stronger effect seen in those with lower birth weights (fig 4). This pattern was less distinct in women. The interaction term for percentage of adult height achieved by age 7 with birth weight was significant in men $(\mathrm{P}=0.003)$ but not in women $(\mathrm{P}=0.82)$. In men, the relation between percentage adult height achieved by age 7 and adult obesity also varied by maternal weight or body mass index, with a stronger relation seen in cohort members with lighter or

Table 2 Standard deviation score of body mass index in groups (thirds) ${ }^{*}$ of birth weight in grams and body mass index in members of the 1958 British birth cohort at 7, 11, 16, 23, and 33 years. Values are mean standard deviation scores of body mass index (95\% confidence interval)

\begin{tabular}{|c|c|c|c|c|c|c|}
\hline \multirow[b]{2}{*}{ Age (years) } & \multicolumn{3}{|c|}{ Men } & \multicolumn{3}{|c|}{ Women } \\
\hline & Lowest third & Middle third & Highest third & Lowest third & Middle third & Highest third \\
\hline 7 & $-0.16(-0.20$ to -0.12$)$ & $-0.03(-0.07$ to 0.01$)$ & $0.19(0.15$ to 0.24$)$ & $-0.12(-0.16$ to -0.08$)$ & $-0.04(-0.09$ to 0.00$)$ & $0.17(0.13$ to 0.21$)$ \\
\hline 11 & $-0.13(-0.17$ to -0.08$)$ & $-0.03(-0.07$ to 0.01$)$ & 0.18 (0.14 to 0.23$)$ & $-0.13(-0.17$ to -0.08$)$ & $0.00(-0.08$ to 0.14$)$ & 0.17 (0.13 to 0.22$)$ \\
\hline 16 & $-0.11(-0.16$ to -0.07$)$ & $-0.02(-0.06$ to 0.03$)$ & $0.16(0.11$ to 0.20$)$ & $-0.07(-0.12$ to -0.02$)$ & $-0.02(-0.07$ to 0.03$)$ & 0.11 (0.06 to 0.16$)$ \\
\hline 23 & $-0.07(-0.12$ to -0.02$)$ & $-0.04(-0.08$ to 0.00$)$ & 0.13 (0.08 to 0.18$)$ & -0.01 (0.06 to 0.03$)$ & $-0.03(-0.08$ to 0.02$)$ & 0.07 (0.03 to 0.12$)$ \\
\hline 33 & $-0.05(-0.10$ to 0.00$)$ & $-0.02(-0.07$ to 0.02$)$ & $0.10(0.05$ to 0.15$)$ & $-0.04(-0.09$ to 0.01$)$ & $-0.04(-0.09$ to 0.01$)$ & 0.09 (0.04 to 0.14$)$ \\
\hline
\end{tabular}

${ }^{*}$ Tertile cut offs for birth weight: $1 \mathrm{st}$ tertile $=3170 \mathrm{~g}$ in males and $3060 \mathrm{~g}$ in females; 2 nd tertile $=3630 \mathrm{~g}$ in males and $3460 \mathrm{~g}$ in females. 

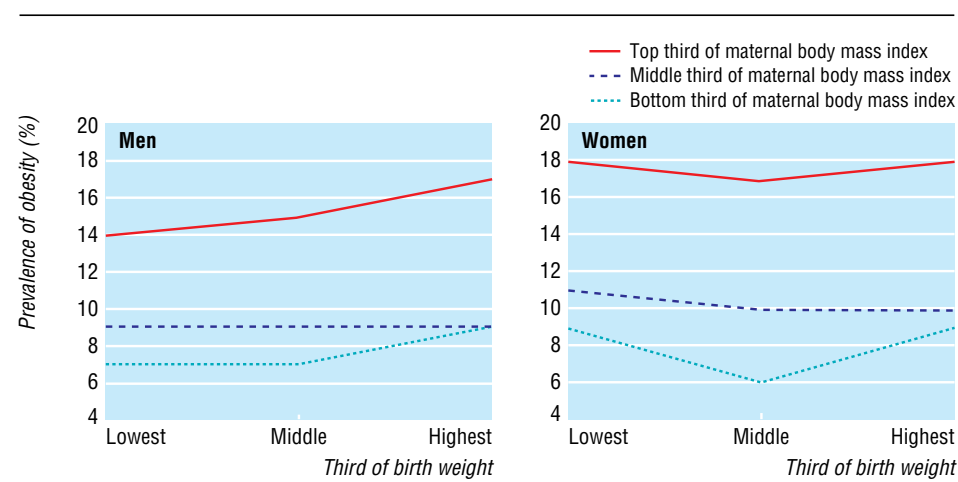

Fig 3 Prevalence of obesity at age 33 by mother's body mass index in 1958 and birth weight in the 1958 British birth cohort. Cut offs for tertiles at 3170 and $3630 \mathrm{~g}$ in men and 3060 and $3460 \mathrm{~g}$ in women

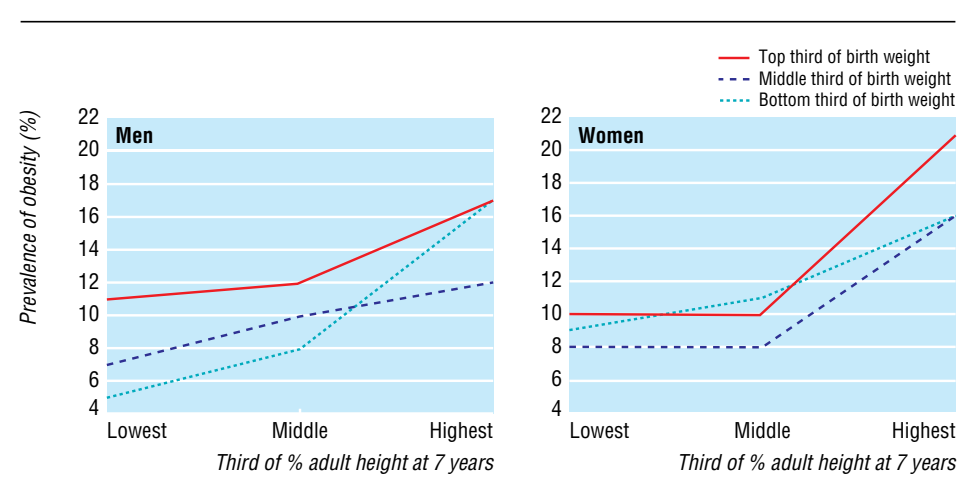

Fig 4 Prevalence of obesity at age 33 by birth weight and percentage adult height at age 7 in the 1958 British birth cohort

thinner mothers. The interaction term for childhood growth with maternal weight on obesity at age 33 was significant in men $(\mathrm{P}=0.04)$, but not in women $(\mathrm{P}=0.26)$. In analyses using body mass index as a continuous outcome, these relations for growth in childhood were also observed, but were less noticeable.

\section{Discussion}

The relation between birth weight and fatness has been investigated in many studies covering a wide age range, but comparisons are difficult owing to different definitions and methods of analysis. Data from the 1958 cohort allowed us to assess the relation at several time points within the same population. Because there is extensive information about the cohort members and their parents, we were able to investigate the issue of confounding factors, which is an important gap in the literature. We found a positive association between the cohort member's birth weight and body mass index which seems to reflect the mother's weight or body mass index.

Other factors that we considered did not affect the relation between birth weight and body mass index. Fetal growth, estimated in terms of birth weight in combination with parental body size, was unrelated to adult obesity, but lighter babies were at a greater risk of obesity if they achieved a greater, rather than a smaller, percentage of their adult height by age 7 . Assessing fetal growth is complex, and there is little consensus on which measure should be used. Different measures may be appropriate for different subsequent health outcomes, and these measures may further differ by sex. ${ }^{20}$ Measures such as length at birth, ponderal index, and head and abdominal circumferences may also need to be considered in addition to those used in this study.

In our data, an increase in birth weight was associated with an increase in body mass index, but the relation became less linear and more $\mathrm{J}$ shaped with increasing age. This is consistent with the literature- to our knowledge there are no reports of a J shaped relation in childhood, whereas several studies report a J shaped relation for fatness in participants aged 17 or older. ${ }^{4-8}$ It is uncertain why the nature of the relation changes over time. Some evidence exists for compensatory growth among individuals with low birth weight, ${ }^{21}$ which would result in an increased body mass index, but this trend needs to be confirmed.

\section{Confounding factors}

Few studies have investigated whether the relation between birth weight and body mass index is confounded by other factors, although those most commonly mentioned in the literature are parental fatness, gestational age, and socioeconomic status. To our knowledge, we are the first group to consider a wide range of factors, particularly maternal and paternal body size. In our study, the relation between birth weight and adult body mass index was largely accounted for by maternal weight or body mass index, and consequently the relation attributed to the intrauterine environment may reflect the mother-child relations in weight and body mass index. Father's height, weight, or body mass index had no influence on the relation between birth weight and adult body mass index. This may be due to paternal body size being less strongly related to the birth weight of the child than maternal size, or it may be because the fathers' heights and weights, as reported by the mothers, were less accurately measured.

Gestational age, social class, parity, mother's age, and mother's smoking habits, which could account for the relation between birth weight and body mass index, had no influence on the relation at age 33.

\section{Intrauterine environment}

There was a weak relation between birth weight and body mass index, which was consistent for men and women, that seems to reflect the tendency of heavier mothers to have heavier babies who subsequently become heavier adults. This is unsurprising and is not very informative in respect of the intrauterine environment and the development of obesity.

Since recent work has found that fetal growth is an important determinant of adult disease, we attempted to investigate whether those babies who did not reach their growth potential in utero (as estimated by birth weight related to parental body size or own adult height) were more likely to become obese adults. We found no evidence that this was the case. Small babies born to tall or heavy parents, or who themselves became tall as adults, did not show a higher prevalence of adult obesity than large babies with tall or heavy parents or who became tall adults. There was no effect on obesity when birth weight was linked to maternal height-this combination of measures might best indicate whether fetal growth was constrained. Our results do not support an effect of fetal growth on adult obesity. 


\section{Childhood growth}

The effect of fetal growth may depend on subsequent patterns of growth in childhood. It is already known that faster rates of maturation, as indexed by various measures, including stage of puberty and adiposity rebound, are associated with increased risk of fatness, ${ }^{2}$ but such studies tend to neglect growth in utero. In the 1958 British birth cohort, we found that although children who achieved more of their adult height between birth and age 7 tended to be heavier rather than lighter at birth, the positive relation between linear growth and adult obesity was strongest in those who were light at birth, at least in men. Thus, men with a lower birth weight who achieved more adult height by age 7 had a risk of obesity comparable with that for men with higher birth weights. Among those who achieved less of their adult height by age 7, the risk of obesity differed by birth weight. The combination of slower growth in utero with achievement of greater proportion of adult height in childhood increases the risk of obesity.

We are unable to distinguish whether prenatal or postnatal factors account for the effect of childhood growth or for the modifying effect of birth weight. However, these findings are potentially important because the combination of low birth weight and rapid linear growth has been identified as a risk for other cardiovascular risk factors, such as increased blood pressure. ${ }^{22}$ For men, the relation between childhood growth and adult obesity varied by mother's weight or body mass index as well as by birth weight, with a stronger positive association in those men whose mother had a lower weight or body mass index.

We cannot explain why birth weight and childhood growth are related to the development of adult obesity in men whereas the pattern in women is less distinct. Maternal weight seems to account for the relation between birth weight and body mass index in both men and women, and the rate of maturation is related to adiposity in both sexes. ${ }^{2}$ There are well known sex differences in fetal growth, and other work has noted sex differences in aspects of fetal and childhood growth and adult disease - the risk of coronary heart disease is increased in women who were short at birth, but caught up in height later in life, and in men who were thin at birth and who caught up in weight later in life. $^{20}$ Several hypotheses for why compensatory growth might be associated with adverse health outcomes in later life have been advanced, ${ }^{20}{ }^{23}$ some of which derive from animal studies, but so far they remain speculative, and the mechanisms involved in humans are largely undefined.

Contributors: TP and CP developed the hypotheses for the study $\mathrm{TP}, \mathrm{CP}$, and $\mathrm{OM}$ discussed core ideas, participated in the analyses, and wrote this paper. TP and CP are guarantors for the study.

Funding: These analyses were funded by the Department of Health. The views expressed in this publication are those of the authors and not necessarily those of the sponsors.

Competing interests: None declared.

1 Dietz WH. Critical periods in childhood for the development of obesity. Am J Clin Nutr 1994;59:955-9.

2 Parsons TJ, Power C, Logan S, Summerbell CD. Childhood predictors of adult obesity: a systematic review. Intl J Obesity 1999;23(suppl 8):S1-107.

3 Whitaker RC, Dietz WH. Role of the prenatal environment in the development of obesity. J Pediatr 1998;132:768-76

4 Seidman DS, Laor A, Gale R, Stevenson DK, Danon YL. A longitudinal study of birth weight and being overweight in late adolescence. Am J Dis Child 1991;145:782-5.

\section{What is already known on this topic}

Birth weight has been shown to be positively related to subsequent fatness

Few studies have investigated whether this relation is confounded by other factors, such as parental size

Birth weight may be an inadequate indicator of the intrauterine environment

\section{What this study adds}

The relation between birth weight and adult body mass index was largely accounted for by mother's weight

Fetal growth indexed by birth weight relative to parental body size was unrelated to adult obesity

Rapid linear growth in childhood increased the risk of obesity in adulthood, especially in males with low birth weight

Among boys who grew rapidly, the risk of obesity in adulthood was similar for both lower and higher birth weights

5 Hulman S, Kushner H, Katz S, Falkner B. Can cardiovascular risk be predicted by newborn, childhood, and adolescent body size? An examination of longitudinal data in urban African Americans. J Pediatr 1998;132:90-7.

6 Curhan GC, Chertow GM, Willett WC, Spiegelman D, Colditz GA, Manson JE, et al. Birth weight and adult hypertension and obesity in women. Circulation 1996;94:1310-5.

7 Curhan GC, Willett WC, Rimm EB, Spiegelman D, Ascherio AL, Stampfer MJ. Birth weight and adult hypertension, diabetes mellitus, and obesity in US men. Circulation 1996;94:3246-50.

8 Fall CH, Osmond C, Barker DJ, Clark PM, Hales CN, Stirling Y, et al. Fetal and infant growth and cardiovascular risk factors in women. $B M J$ 1995;310:428-32

9 Leon DA, Koupilova I, Lithell HO, Berglund L, Mohsen R, Vagero D, et al. Failure to realise growth potential in utero and adult obesity in relation to blood pressure in 50 year old Swedish men. BMJ 1996;312:401-6.

10 Van Lenthe FJ, Kemper CG, van Mechelen W. Rapid maturation in adolescence results in greater obesity in adulthood: the Amsterdam growth and health study. Am J Clin Nutr 1996;64:18-24.

11 Freeman JV, Power C, Rodgers B. Weight-for-height indices of adiposity: relationships with height in childhood and early adult life. Intl J Epidemio 1995;24:970-6.

12 Beunen G, Malina RM, Lefevre J, Claessens AL, Renson R, Simons J, et al. Size, fatness and relative fat distribution of males of contrasting maturity status during adolescence and as adults. Intl J Obesity 1994:18:670-8.

13 Centre for Longitudinal Studies Institute of Education. National Child Development Study Composite File including selected Perinatal Data and sweeps one to five [computer file]. National Birthday Trust Fund, National Children's Bureau, City University Social Statistics Research Unit [original data producers]. Colchester: The Data Archive distributor (1994) SN: 3148. 2000.

14 Butler NR, Bonham DG. Perinatal mortality. Edinburgh: Churchill Livingstone, 1963.

15 Ferri E. Life at 33: the fifth follow-up of the national child development study London: National Children's Bureau, 1993

16 Power C, Moynihan C. Social class and changes in weight-for-heigh between childhood and early adulthood. Intl J Obesity 1988;12:445-53.

17 Lake JK. Body size in child and adulthood: implications for adult health [dissertation]. London: University College London, 1998.

18 Clinical guidelines on the identification, evaluation, and treatment of overweight and obesity in adults: executive summary. Expert panel on the identification, evaluation, and treatment of overweight in adults. Am J Clin Nutr 1998;68:899-917.

19 Great Britain General Registrar's Office. Census 1951: classification of occupations. London: HMSO, 1956.

20 Forsen T, Eriksson JG, Tuomilehto J, Osmond C, Barker DJ. Growth in utero and during childhood among women who develop coronary heart disease: longitudinal study. BMJ 1999;319:1403-7.

21 Ong KK, Ahmed ML, Emmett PM, Preece MA, Dunger DB. Association between postnatal catch-up growth and obesity in childhood: prospective cohort study. BMJ 2000;320:967-71. [Published erratum appears in $B M$ J 2000;320:1244.]

22 Huxley RR, Shiell AW, Law CM. The role of size at birth and postnatal catch-up growth in determining systolic blood pressure: a systematic review of the literature. J Hypertens 2000;18:815-31.

23 Eriksson JG, Forsen T, Tuomilehto J, Winter PD, Osmond C, Barker DJ Catch-up growth in childhood and death from coronary heart disease: longitudinal study. BMJ 1999;318:427-31.

(Accepted 29 June 2001) 